\title{
Detecting "Strict" Left Bundle Branch Block from 12-lead Electrocardiogram using Support Vector Machine Classification and Derivative Analysis
}

\author{
Nipun D Perera ${ }^{1}$, Chathuri Daluwatte ${ }^{2}$ \\ ${ }^{1}$ Department of Electronic and Telecommunication Engineering, University of Moratuwa, Sri Lanka \\ ${ }^{2}$ Division of Applied Regulatory Science, Office of Clinical Pharmacology, Center for Drug \\ Evaluation and Research, U.S. Food and Drug Administration, Silver Spring, MD, USA
}

\begin{abstract}
Cardiac Resynchronization Therapy (CRT) is generally indicated for heart failure patients with a left bundle branch block (LBBB). "Strict" $L B B B$ criteria have been proposed as a better predictor of benefit from CRT. Automatic detection of "strict" LBBB criteria may improve outcomes for heart failure patients by reducing high false positive rates in LBBB detection. This study proposes an algorithm to automatically detect "strict" $\angle B B B$, developed and tested using ECGs made available via the International Society of Computerized Electrocardiology (ISCE) LBBB initiative. The dataset consists of 12-lead Holter ECGs recorded before the therapy from the MADIT-CRT clinical trial.

The algorithm consists of multi-lead QRS complex detection using length transform, a support vector machine (SVM) classifier to identify $Q S$ - or $r S$ - configurations and identification of mid-QRS notching and slurring by analyzing the variation of first and second derivatives of the signals respectively. The algorithm achieved an accuracy of $80 \%$, sensitivity of $86 \%$, specificity of $73 \%$, positive predictive value (PPV) of $81 \%$ and negative predictive value of $79 \%$ on the training set. It achieved accuracy, sensitivity, specificity, PPV and NPV of $81 \%$, $88 \%, 75 \%, 79 \%$ and $85 \%$ on the test set. High sensitivity to minor slurring and errors in QRS detection result in low specificity for $L B B B$ detection.
\end{abstract}

\section{Introduction}

Left bundle branch block (LBBB) is the delayed conduction down the left bundle branch which results in delayed contraction of the left ventricle with respect to its right counterpart [1]. This condition leads to dilated cardiomyopathy and widened QRS. Restoration of the left ventricle synchrony is achieved by cardiac resynchronization therapy (CRT). Despite applying this device therapy in approximately 75000 patients per year in the United States, nearly one-third of the patients did not entirely benefit from this since these patients did likely not comply with the requirements of a true LBBB [2]. Hence, "strict" LBBB criteria have been proposed to improve LBBB detection to alleviate the false positive rate in LBBB detection and to improve the efficacy of CRT.

The "strict" LBBB criteria include QRS duration of 140 $\mathrm{ms}$ for male or $130 \mathrm{~ms}$ for female, QS- or rSconfigurations in leads V1 and V2 and mid-QRS notching or slurring in 2 or more leads out of leads I, aVL, V1, V2, V5 and V6 [3]. Slurs/notches were required to have an onset after $40 \mathrm{~ms}$ and before $50 \%$ of the QRS width, relative to the onset of the global QRS complex.

This study proposes an algorithm, combining rule-based methods, machine learning and time-domain signal analysis to detect "strict" LBBB automatically. The algorithm also detects the onset and offset of notches and slurs which is vital for "strict" LBBB detection.

\section{Methodology}

\subsection{Experimental Dataset}

Data from the International Society of Computerized Electrocardiology (ISCE), LBBB initiative were used in this study $[2,4]$ with corrected adjudicated criteria [4]. The dataset consists of 12-lead ECGs sampled at $1 \mathrm{kHz}$ with an amplitude resolution of $3.75 \mu \mathrm{V}$, recorded before CRT using Holter recorders with Mason-Likar lead placement, randomly divided into a training set with 300 records and a test set with 302 records. For each subject, the dataset contained $10 \mathrm{~s}$ ECG signal and a median beat derived from it. For this study, the median beat was used and was smoothed using a Savitzky-Golay filter of polynomial order 3 and frame length of 15.

The algorithm consists of three components: QRS complex detection, QS- or rS- configuration detection and notching/slurring detection. Output of these three components were combined per the "strict" LBBB criteria, to predict presence of LBBB. All signal processing and algorithm development was done in MATLAB (Release 2017b, The MathWorks, Inc., Natick, Massachusetts, United States). 


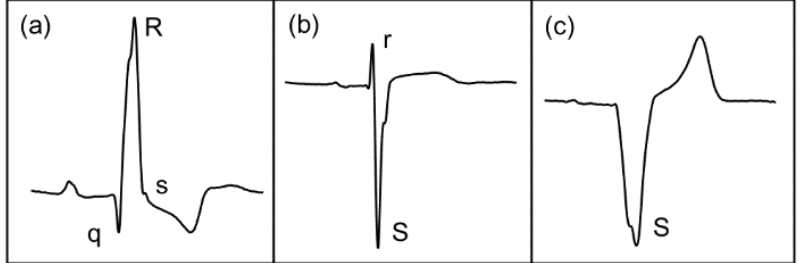

Figure 1. Example QRS morphology on the ECG lead V1 when (a) QS- configuration or rS- configuration are absent, (b) rSconfiguration with small $\mathrm{R}$ wave followed by deep $\mathrm{S}$ wave and (c) QS- configuration characterized by a deep $\mathrm{S}$ wave.

\subsection{QRS Detection}

QRS complex detection was performed using length transform [5] which facilitated multi-lead QRS detection using median beats from all 12 leads. Multi-channel length transform was calculated using equation (1) where $\mathrm{x}_{\mathrm{j}}(\mathrm{t})$ is the median beat of the $\mathrm{j}^{\text {th }}$ ECG lead, with a window size (w) of 70 and a global threshold of 1700. Global threshold was selected to minimize the mean square error between annotated onset and calculated onset $\left(\mathrm{QRS}_{\text {on }}\right)$ and annotated width and calculated width $\left(\mathrm{QRS}_{\mathrm{w}}\right)$ on the training set. The resulting $\mathrm{QRS}_{\text {on }}$ and $\mathrm{QRS}_{\mathrm{w}}$ estimates the global QRS onset and global QRS duration for the 12-lead median ECG beat.

$$
L(w, t)=\int_{t}^{t+w} \sqrt{\sum_{j=1}^{12}\left(\frac{d x_{j}(t)}{d t}\right)^{2}}
$$

\subsection{QS- or rS- Configuration Detection}

"Strict" LBBB criteria dictate that either QS- or rSconfigurations are present in both leads V1 and V2. QS- is characterized by a lone, deep negative wave whereas rS- is characterized by a small $\mathrm{R}$ wave followed by a deep $\mathrm{S}$ wave [6] (Figure 1). A support vector machine (SVM) classifier with radial basis function (RBF) [7] was trained to detect the presence of either of the configurations. The dataset for QS- or rS- detection was prepared using the extracted QRS complex in median beats from leads V1 and V2. Presence of either of the configurations was considered as positive class during training. The SVM classifier was trained using $75 \%$ of randomly selected training set data and was tested using the remaining $25 \%$ to obtain the best configuration with features: maximum/minimum amplitude, mean signal power and the signal entropy [8] of the QRS complex. Signal entropy $(\mathrm{H})$ of QRS complex $\mathrm{y}_{\mathrm{k}}(\mathrm{t})$ where $\mathrm{k} \in$ leads $\{\mathrm{V} 1, \mathrm{~V} 2\}$ was calculated by generating the histogram of amplitudes with $\mathrm{n}$ number of bins of $100 \mu \mathrm{V}$ width where $\mathrm{p}_{\mathrm{i}}$ is the frequency of occurrence of $\mathrm{i}^{\text {th }}$ bin of amplitudes, followed by substitution to equation 2 .

$$
\left[p_{1}, p_{2}, \ldots . p_{n}\right]=\operatorname{hist}\left\{y_{k}(t)\right\}
$$

$$
H=\sum_{i=1}^{n} p_{i} \log _{2} p_{i}
$$

\subsection{Notching/Slurring Detection}

Detection of mid-QRS notching in leads I, aVL, V1, V2, V5 and V6 was performed using the number of inflection points $\left(\mathrm{M}^{\prime}\right)$ present during the QRS duration calculated in section 2.2. Inflection points were detected as the points where first derivative of the median beat crosses zero. When one or more notches are present, the number of zerocrossing points on the first derivative within the QRS complex is equal to or greater than 3 (Figure 2a). Therefore, the onset and offset of first notch corresponds to the $1^{\text {st }}$ and $3^{\text {rd }}$ inflection point, the onset and offset of second notch corresponds to the $3^{\text {rd }}$ and $5^{\text {th }}$ inflection point and so on.

During notching, the signal is not strictly increasing or decreasing. However, during slurring, the signal is either strictly increasing or decreasing with a distinct change of slope, which can be detected using zero-crossings in the second derivative (Figure 2b). Therefore, slurs were identified using the number of inflection points of the second derivative $\left(\mathrm{M}^{\prime \prime}\right)$ when notches are not present $\left(\mathrm{M}^{\prime}<\right.$ 3 ), in a piecewise manner as shown in equation 3 where $\mathrm{QRS}_{\text {on }}$ and $\mathrm{QRS}_{\text {off }}$ are detected onset and offset of QRS complex and $\mathrm{T}$ is the time corresponding to inflection point. The onset and offset of the slurs were determined the same manner it was done for the notches, but with zerocrossing detection on second derivative.

$$
\begin{gathered}
{\left[Q R S_{\text {on }}, T_{1}\right] \text { and }\left[T_{1}, Q R S_{\text {off }}\right] \text { for } M^{\prime}=1} \\
{\left[Q R S_{\text {on }}, T_{1}\right]\left[T_{1}, T_{2}\right] \text { and }\left[T_{2}, Q R S_{\text {off } f}\right] \text { for } M^{\prime}=2}
\end{gathered}
$$

Once the slurs and/or notches were detected, it was checked whether the onset satisfies the "strict" LBBB criteria by checking $Q R S_{\text {on }}+40 \leq t_{\text {on }} \leq Q R S_{\text {on }}+$ $Q R S_{w}$ where $t_{\text {on }}$ is the onset of notch or slur.
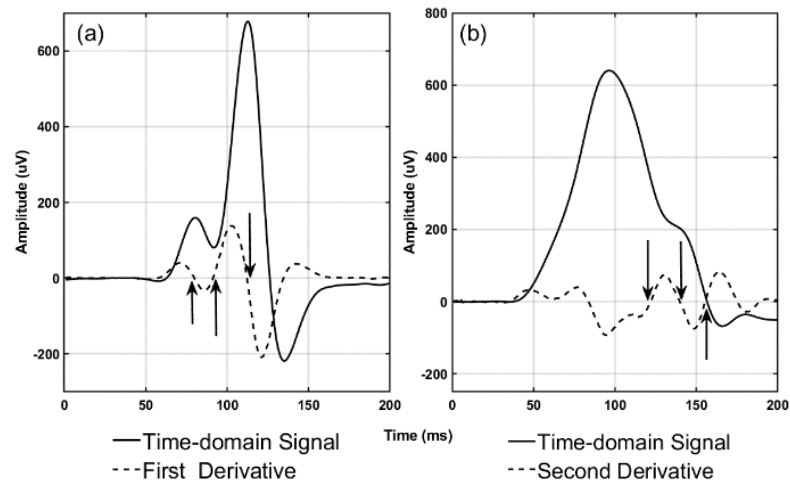

Figure 2. (a) First derivative of QRS complex on lead V1 during a notch, which satisfies notch criterion: number of inflection points $\left(M^{\prime}\right)$ equals 3. (b) Second derivative of QRS complex on lead V5 which satisfies slur criterion: number of inflection points $\left(M^{\prime \prime}\right)$ equals 3 . 


\subsection{Statistical Analysis}

The "strict" LBBB detection performance of the algorithm and SVM classifier performance to detect QSor rS- configuration were assessed using accuracy (Acc), sensitivity (Se), specificity (Sp), positive predictive value (PPV) and negative predictive value (NPV), as per equation 4 and confusion matrix (table 1) when presence of LBBB and presence of QS- or rS- configuration was considered as the positive class (+ve).

$$
\begin{aligned}
& A c c=\frac{T P+T N}{\text { Total }} \quad S e=\frac{T N}{T P+F N} \quad S p=\frac{T N}{T N+F P} \\
& P P V=\frac{T P}{T P+F P} \quad N P V=\frac{T N}{T N+F N}
\end{aligned}
$$

Table 1. Confusion matrix.

\begin{tabular}{|c|c|c|}
\hline \multirow{3}{*}{ 胥 } & $+v e$ & -ve \\
\hline & $\begin{array}{l}\text { TP: No. of +ve's } \\
\text { classified as +ve }\end{array}$ & $\begin{array}{l}\text { FN: No. of +ve's } \\
\text { classified as -ve }\end{array}$ \\
\hline & $\begin{array}{l}\text { FP: No. of +ve's } \\
\text { classified as -ve }\end{array}$ & $\begin{array}{l}\text { TN: No. of -ve's } \\
\text { classified as -ve }\end{array}$ \\
\hline
\end{tabular}

Classified

The QRS detector performance was assessed using two metrics: difference between calculated onset and annotated onset $(\Delta s)$ and difference between measured and annotated QRS width $(\Delta \mathrm{d})$. The deviance from annotations was tested using one tailed t-test with null hypothesis: the difference is 0 with type I error set to 0.05 . All statistical analysis was performed in Minitab ${ }^{\circledR}$ Statistical Software (Minitab 18, Minitab, Inc., State College, Pennsylvania, USA).

\section{Results}

The "strict" LBBB detection performance of the algorithm is summarized in table 2. The algorithm has similar performance metrics for initiative provided training and test datasets, which suggests that the rule-based approach is consistent. The algorithm performed well in terms of sensitivity but, relatively poorly on specificity due to false positives. However, the algorithm has good positive and negative predictive values on both datasets.

Table 2. LBBB detection performance.

\begin{tabular}{cccccc}
\hline \multirow{2}{*}{ Dataset } & \multicolumn{5}{c}{ Performance (\%) } \\
\cline { 2 - 6 } & Acc & Se & Sp & PPV & NPV \\
\hline Training & 80 & 86 & 73 & 81 & 79 \\
\hline Test & 81 & 88 & 75 & 79 & 85 \\
\hline
\end{tabular}

The performance of the SVM classifier used to detect QS- or rS- configurations is summarized on table 3. The specificity and NPV on the test set indicates that the classifier has falsely detected $16 \%$ of QS- or rSconfigurations. On both datasets the QRS onset was detected early $(\Delta \mathrm{s}=-1.8 \pm 8.9 \mathrm{~ms}, \mathrm{p}=0.001$ on the training set, $\Delta \mathrm{s}=-1.3 \pm 9.2 \mathrm{~ms}, \mathrm{p}=0.015$ on the test set) and calculated QRS width was wider $(\Delta \mathrm{d}=8.2 \pm 18.0 \mathrm{~ms}, \mathrm{p}<$ 0.0001 on the training set, $\Delta \mathrm{d}=8.8 \pm 19.0 \mathrm{~ms}, \mathrm{p}<0.0001$ on test set). These results suggest that errors in QRS detection and QS- or rS- detection can directly contribute to high false positives in LBBB detection. Errors in QRS detection can indirectly contribute to false positive LBBB detection as well by affecting the slur detection.

Table 3. QS- or rS- configuration detection performance.

\begin{tabular}{cccccc}
\hline \multirow{2}{*}{ Dataset } & \multicolumn{5}{c}{ Performance (\%) } \\
\cline { 2 - 6 } & Acc & Se & Sp & PPV & NPV \\
\hline Training & 98 & 100 & 92 & 98 & 98 \\
\hline Test & 96 & 98 & 84 & 97 & 89 \\
\hline
\end{tabular}

\section{Discussion}

"Strict" criteria for LBBB have been proposed to better predict patient benefit from CRT therapy. This study presents an algorithm to automatically detect the presence of "strict" LBBB on 12 lead Holter ECGs. The algorithm analyses the median beat combining rule-based methods with machine learning. The algorithm has performed well on sensitivity but relatively poorly on specificity which elicits high false positives.

Errors caused by QRS onset detection and QRS duration calculation affect the LBBB detection when determining whether the onset of a slur/notch is within the specified region per "strict" criteria. For example, Figure 3 shows how a notch was missed due to late detection of QRS onset by $8 \mathrm{~ms}$ which caused the notch onset to be detected before $40 \mathrm{~ms}$ with respect to detected QRS onset and hence, do not comply with notch/slur onset criterion. In this scenario, the calculated notch onset deviated from the annotated onset only by $1 \mathrm{~ms}$.

The slur detector is sensitive to minor slurring and/or artefacts which were not identified as slurs during annotation. The slur detector developed in this study adjudicated that the QRS complex contained a slur, if the

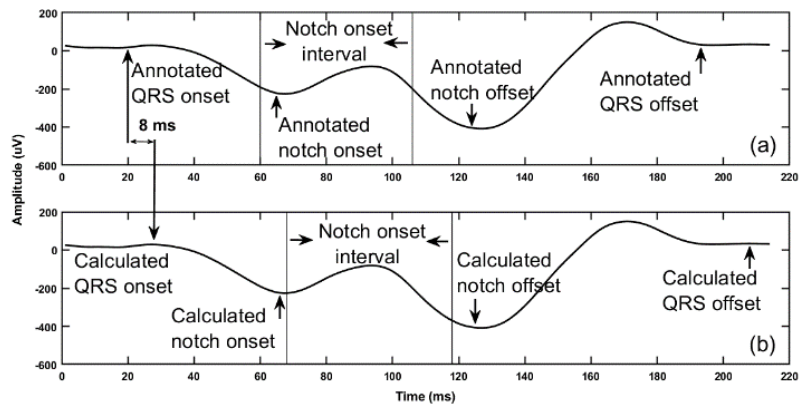

Figure 3. An example of a missed notch detection due to late detection of QRS. (a) Annotated QRS complex and notch (b) Detected QRS complex and notch, $\Delta \mathrm{s}=8 \mathrm{~ms}$ and $\Delta \mathrm{d}=$ $16 \mathrm{~ms}$, thus notch onset is out of bound. 


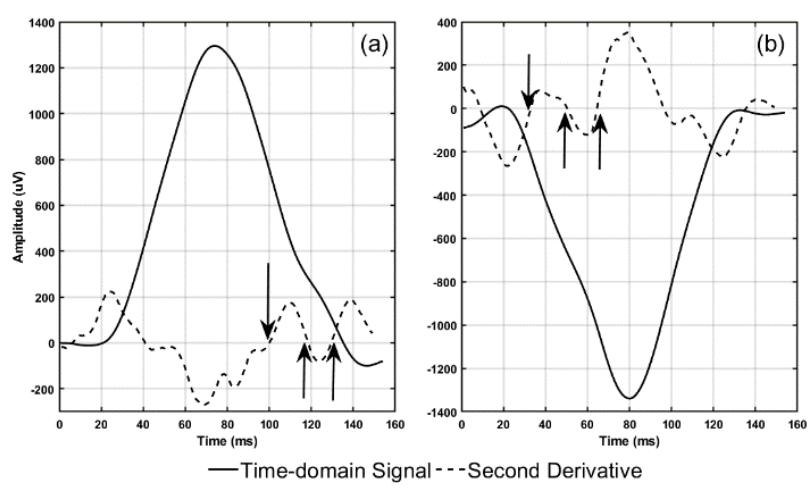

Figure 4. Examples of slur detection by algorithm: 3 zerocrossing points on second derivative of QRS complex (indicated with arrows) whereas (a) is annotated as a slur and (b) is not annotated as a slur and could resemble an artefact.

second derivative contained three zero-crossing points, regardless of how prominent the slur was (the degree to which slurring occurs) which is further explained in Figure 4. This compromised the specificity and the positive predictive value of the algorithm by falsely detecting slurs.

Even though the onset and offset of a notch could be clearly perceived, it is ambiguous and not clearly defined for a slur. In the literature, slurring is usually indicated as a point [9] rather than a duration with a clearly defined onset, but our algorithm detected onset and offset of slurring in an ad-hoc manner using zero-crossing points of the second derivative.

\section{Conclusion}

We developed an automatic "strict" LBBB detection algorithm using the time domain behavior of the median beat, its first derivative and second derivative. The algorithm has performed well in identifying "strict" LBBB cases but has a considerable number of false positives. The algorithm detected wide QRS complexes early and QS- or rS- configurations falsely, which caused false positives. Improving QRS detection, QS- or rS- configuration and slur detection can further improve LBBB detection. More clear definition of slurs in terms of the onset and offset would facilitate such improvements.

\section{Disclaimer}

This article reflects the views of the authors and should not be construed to represent FDA's views or policies. The mention of commercial products, their sources, or their use in connection with material reported herein is not to the construed as either an actual or implied endorsement of such products by the Department of Health and Human Services.

\section{Acknowledgements}

This project was supported in part by a research fellowship from the Oak Ridge Institute for Science and Education through an interagency agreement between the U.S. Department of Energy and the FDA. The authors would like to thank the organizers of the LBBB initiative.

\section{References}

[1] LBBB. https://en.ecgpedia.org/wiki/LBBB, August 2012. (Accessed on 06/04/2018).

[2] Guidelines for the LBBB Initiative of the ISCE 2018 meeting. http://thew-project.org/LBBB Initiative.htm. (Accessed on 05/04/2018).

[3] Strauss DG, Selvester RH, Wagner GS. Defining left bundle branch block in the era of cardiac resynchronization therapy. American Journal of Cardiology. 2011 Mar 15;107(6):92734.

[4] Zusterzeel R, Vicente J, Ochoa-Jimenez R, Zhu J, Couderc JP, Akinnagbe-Zusterzeel E, Strauss DG. Computerized Electrocardiology ECG Initiative for the Automated Detection of Strict Left Bundle Branch Block. Journal of Electrocardiology. 2018. doi: 10.1016/j.jelectrocard.2018.08.001 (article in press).

[5] Gritzali F. Towards a generalized scheme for QRS detection in ECG waveforms. Signal processing. 1988 Sep $1 ; 15(2): 183-92$

[6] Gertsch M. The Normal ECG and its (Normal) variants. In The ECG 2004 (pp. 19-43). Springer, Berlin, Heidelberg.

[7] Hearst MA, Dumais ST, Osuna E, Platt J, Scholkopf B. Support vector machines. IEEE Intelligent Systems and their applications. $1998 \mathrm{Jul} ; 13(4): 18-28$.

[8] Perera ND, Madarasingha C, De Silva AC. Spatial Feature Reduction in Long-term EEG for Patient-specific Epileptic Seizure Event Detection. In Proceedings of the 9th International Conference on Signal Processing Systems 2017 Nov 27 (pp. 230-234). ACM.

[9] Heng SJ, Clark EN, Macfarlane PW. End QRS notching or slurring in the electrocardiogram: influence on the definition of "early repolarization". Journal of the American College of Cardiology. 2012 Sep 4;60(10):947-8.

Address for correspondence:

Nipun Dilesh Perera

Department of Electronic and Telecommunication Engineering, University of Moratuwa, Sri Lanka.

130441f@uom.lk 